\title{
Allosteric modulators in CNS disorders: review
}

\begin{abstract}
A variety of central nervous system disorders were treated using $\mathrm{G}$ protein-coupled receptors (GPCRs) modulators. The discovery of allosteric modulators of GPCRs offers novel treatments with added benefits compared to orthosteric site modulators of GPCRs. The target site for allosteric modulators is separate from the orthosteric site and is still able to modulate the receptor function. Allosteric modulators either potentiate or inhibit the receptor response, and provide much greater subtype selectivity those orthostatic agents for the same receptors. Experiments revealed Positive Allosteric Modulators show allosteric agonism when used in combination with an endogenous ligand. Allosteric modulators permit increased drug selectivity and demonstrate a significant decrease in adverse effects. Studies showed the potential of some allosteric modulators of GPCRs and AMPA receptors in multiple Central Nervous System disorders including Alzheimer's and Parkinson's disease.
\end{abstract}

Keywords: allosteric modulation, orthosteric modulation, bitopic binding, Schizophrenia, AMPA receptor modulation
Volume 9 Issue $6-2018$

\author{
Roy Sebastian \\ Baylor Scott \& White Medical Center, USA
}

Correspondence: Roy Sebastian, BSW Medical CenterSunnyvale, 23I S.Collins Rd., Sunnyvale, TX 75|82, USA, Tel (2I4) 9307527,Email roy.sebastean@usat.edu

Received: April 05, 2017 | Published: November 15, 2018
Abbreviations: GPCRs, G Protein-Coupled Receptors; PAM, Positive Allosteric Modulators, NAM, Negative Allosteric Modulators; SAMs, Silent Allosteric Modulators, NMDA, N-Methyl-D-Aspartate, mGlu, metabotropic glutamate, mAChRs, muscarinic Acetylcholine Receptors, PLD, phospholipase D, DOI, 2,5-dimethoxy-4-iodoamphetamine, AMPA, (2-amino-3-(3-hydroxy5-methyl-isoxazol-4-yl) propanoic acid

\section{Introduction}

The discovery and development of new therapeutic agents are challenging for all therapeutic areas related to central nervous system disorders due to very high attrition rate. ${ }^{1}$ Many current clinical therapeutic agents act by targeting GPCRs and related signaling pathways. ${ }^{2}$ While many available drugs on the market target GPCR signaling pathways, overall less than $20 \%$ of GPCRs are targeted. ${ }^{3}$ The research for newer drugs targeting GPCRs focused on the developing traditional agonists and antagonists agents that target the orthostatic neurotransmitter binding site. While this has been effective, there are many instances where the high conservation of the orthostatic binding site across related receptors prevents the development of selective subtype agents. In recent years, advances in the development of allosteric modulators of GPCRs have emerged as promising new approaches for developing therapeutic agents that may be useful for the treatment of CNS disorders.

The binding site of allosteric modulators are separate from the orthosteric binding site of the endogenous ligand and are usually less highly conserved than the orthosteric site. ${ }^{2}$ This characteristic has allowed optimization of allosteric modulators to achieve higher subtype selectivity than traditional orthosteric ligands. Other potential advantages of the allosteric modulators are the ability to develop agents that have functional selectivity, leading to potential targeting of distinct downstream signaling pathways, and a greater diversity of chemical scaffolds that can facilitate optimization of pharmacokinetic properties of potential drug candidates. Increase in the development of allosteric agents showed a various range of drug activities, including PAMs and NAMs, and silent allosteric modulators (SAMs) that bind to the allosteric site but do not potentiate or inhibit the action of endogenous agonist. ${ }^{4}$ Also, allosteric agonists with a bitopic binding mode (binds to both the allosteric site and the orthosteric site) have been identified. ${ }^{5}$ Many allosteric modulators are showing promise for potential treatment of CNS diseases. Among these, allosteric modulators of the metabotropic glutamate (mGlu) receptors and the muscarinic acetylcholine receptors (mAChRs) are the most advanced and well-studied ones. Allosteric modulators of particular subtypes of mGlu receptors have a great scope in the treatment of schizophrenia, autistic spectrum disorders, and Parkinson's disease. ${ }^{2}$ Positive allosteric modulators of the M1 and M4 muscarinic receptors demonstrate promising applications in both Alzheimer's disease and schizophrenia. Several of these allosteric modulator candidates moving from preclinical models into clinical development. ${ }^{6}$ AMPA (2-amino-3-(3-hydroxy-5-methyl-isoxazol-4-yl) propanoic acid) receptors mediate fast excitatory synaptic transmission at $\sim 70-80$ $\%$ of brain synapses. Potentiating the activity of AMPA receptors may improve cognitive deficits in disorders such as schizophrenia in which glutamatergic transmission is weakened and impaired. AMPA receptor positive allosteric modulators may have beneficial actions in managing CNS disorders. Schizophrenia is a psychiatric disorder affecting approximately $1 \%$ of the population across the globe. ${ }^{7}$ The manifestation of Schizophrenia includes a triad of symptom clusters: positive symptoms (psychosis), negative symptoms, and cognitive impairment. Current therapies for the treatment of psychosis in schizophrenia patients focus on the blockade of the D2 dopamine receptors. Low efficacy and serious adverse side effects such as extrapyramidal motor symptoms and metabolic syndrome and sexual dysfunction limit the treatment using D2dopamine antagonists. It limits the successful therapeutic window for the D2 antagonists, and some D2 antagonists only show partial efficacy in some patients. Some patients often switch medications for better symptom management. NMDA receptor channel blockers, including Phencyclidine, ketamine, and dizocilpine (MK-801), induce psychosis in human volunteers. Studies show that Group II (mGlu2 and mGlu3) agonists or mGlu2 PAMs are capable of controlling the positive symptoms, while mGlu5 PAMs have efficacy in reducing all symptoms clusters schizophrenia 
patients. ${ }^{8}$ Activation of mGlu4 with positive allosteric modulators is a promising therapeutic area for management of schizophrenia and development of newer antipsychotic medications. The Group III mGlu4-preferring orthosteric agonist LSP1-2111 demonstrated a dose-dependent inhibition of amphetamine-induced hyperactivity and reversal of MK-801 induced deficits in novel object recognition in rats. ${ }^{9}$ This indicates the potential effects of LSP1-2111 on both the positive and cognitive symptoms of schizophrenia. Further, studies in animal models with mGlu4 PAMs showed promising antipsychotic effects. ${ }^{10}$ For example, the brain-penetrant mGlu4 PAMs Lu AF21934 and Lu AF32615 showed dose-dependent reduction of amphetamine-induced hyperactivity and antagonism of 2,5-dimethoxy-4-iodoamphetamine (DOI)-induced head twitch tests in wild-type mice supporting a key role for mGlu4 in brain circuits involved in these behavioral models. ${ }^{11}$ It did not have any effect in mGlu4 $-/-$ mice. Some clinical and preclinical studies have led to the hypothesis that reduced the activity of NMDA subtypes of glutamate receptors or brain circuits that are regulated by NMDA receptors may play an important role in the pathophysiology of underlying schizophrenia. ${ }^{12}$ Studies suggest that mGlu5 PAMs have the potential to provide a fundamental advance in schizophrenia therapy that could have efficacy in the treatment of all major symptoms of the disease. ${ }^{12}$ Modulation of the glutamatergic function using mGlu5 PAMs causes less adverse effects than the treatment using direct agonists of mGlu5 or NMDA receptors. Recent studies reveal that some mGlu5 PAMs induce severe seizure activity ${ }^{13}$ and excitotoxicity leading to cell death in the auditory cortex, hippocampus, and other forebrain regions. mGlu5 PAMs and other GPCR allosteric modulators can regulate specific aspects of mGlu5 signaling without affecting others. ${ }^{14}$ Recent studies demonstrate that hepatic metabolism of certain mGlu5 PAMs produces metabolites that have substantial activities that are different from the activity of the parent compound, and that can contribute to the adverse effects. ${ }^{12}$

\section{Future opportunities}

Allosteric modulators of GPCRs represent promising drug candidates for the treatment of multiple CNS disorders. In addition to the metabotropic and muscarinic allosteric modulators, drugs have been developed targeting a number of other GPCRs. Allosteric modulators are also developed for enzymes including allosteric kinase (Akt) inhibitors and phospholipase D (PLD) in addition to GPCRs. ${ }^{15}$ There are many advantages of allosteric modulators of GPCRs including subtype selectivity and functional selectivity. With a deeper understanding of signaling bias, it may be possible to avoid undesirable side effects elicited by parallel signaling pathways by targeting specific downstream receptor pathways. Due to the functional selectivity possible with allosteric ligands, it is crucial to incorporate multiple functional assays into drug screening paradigms. An increase in efforts to develop allosteric modulators of GPCRs and the collaboration between industry and academics offers a promising picture for the effort to develop effective treatments for CNS diseases.

\section{AMPA receptor modulation}

AMPA (2-amino-3-(3-hydroxy-5-methyl-isoxazol-4-yl) propanoic acid) receptors mediate fast excitatory synaptic transmission at $\sim 70$ $80 \%$ of brain synapses. AMPA receptors are permeable to $\mathrm{Na}+$ and activation of these receptors results in postsynaptic depolarization. ${ }^{16}$ The density of AMPA receptors in the post-synaptic active zone determines the synaptic strength, i.e., the current density in response to a packet of glutamate release into the synapse. The rapid insertion or removal of AMPA receptors within the active zone is a principal mechanism for regulating synaptic strength. The basis of NMDA receptor-regulated long-term potentiation and depression (LTP and LTD) is the dynamic regulation of AMPA receptor trafficking which is widely held to be the molecular basis for some forms of learning and memory. ${ }^{17}$ A minority of AMPA receptors are permeable to both $\mathrm{Na}+$ and $\mathrm{Ca} 2+$. These $\mathrm{Ca} 2+$ permeable AMPA receptors directly regulate intracellular $\mathrm{Ca} 2+$ signaling cascades in some synaptic locations. Potentiating the activity of AMPA receptors may improve cognitive deficits in disorders such as schizophrenia in which glutamatergic transmission is weakened and impaired. AMPA receptor positive allosteric modulators may have at least three beneficial actions in this regard:

1. Direct facilitation of AMPA receptor-mediated depolarization, mimicking NMDA receptor-mediated AMPA receptor insertion in LTP

2. Increasing depolarization-induced removal of the $\mathrm{Mg} 2+$ block of the NMDA receptor to facilitate the induction of LTP, ${ }^{18}$

3. Induction of activity-dependent BDNF synthesis to augment activation of signaling pathways downstream to this neurotrophic factor. The allosteric sites on AMPA receptors provide a target for amplifying receptor activity without eliminating either signal content or the homeostatic processes that maintain excitatory neurotransmission in the physiological range.

\section{Conclusion}

Allosteric modulators target a site separate from the orthosteric site to modulate GPCRs receptor function. These allosteric agents can either potentiate or inhibit the receptor response and provide much greater subtype selectivity than orthosteric ligands for the same receptors. The drug research for allosteric modulators has offered valuable insight into viable methods for hit-to-lead development. The complex pharmacology of functional bias, mode switching, and bitopic ligand activity complicate the research for allosteric ligands. They also provide more clear insight on the function of GPCRs. After all, functional selectivity may become the foundation for allosteric modulators targeting diseases linked to a specific signaling pathway. Application of appropriate allosteric modulator enhances the traditional drug's potency and permits to use a lower dosage to elicit the same response. It also helps to minimize the adverse effects caused by traditional anti-psychotic drugs. In conclusion, the studies on humans for AMPA PAMs are still very limited and do not provide a clear verdict on the future of these agents in. AMPA PAMs do not appear to be effective antipsychotics when given in monotherapy, while they may have a moderate efficacy on negative and cognitive symptoms when co-administered with conventional antipsychotics. Allosteric modulators require further investigation to identify the appropriate combination of allosteric and orthosteric modulators that can be used more effectively in common psychiatric disorders while minimizing tolerance and other adverse effects.

\section{Acknowledgements}

None.

\section{Conflict of interest}

The author declares that there is no conflict of interest. 


\section{References}

1. Kola I, Landis J. Can the pharmaceutical industry reduce attrition rates? Nat Rev Drug Discov. 2004;3(8):711-715.

2. Nickols HH, Conn PJ. Development of allosteric modulators of GPCRs for treatment of CNS disorders. Neurobiol Dis. 2014;61:55-71.

3. Allen JA, Roth BL. Strategies to Discover Unexpected Targets for Drugs Active at G Protein-Coupled Receptors. Rev Pharmacol Toxicol. 2011;51:117-144.

4. Melancon BJ, Hopkins CR, Wood MR, et al. Allosteric Modulation of Transmembrane Spanning Receptors: Theory, Practice and Opportunities for CNS Drug Discovery. J Med Chem. 2012;55(4):1445-1464.

5. Foster DJ, Jones CK, Conn PJ. Emerging Approaches for Treatment of Schizophrenia: Modulation of Cholinergic Signaling. Discov Med. 2012;14(79):413-420.

6. Foster DJ, Choi DL, Conn PJ, et al. Activation of $\mathrm{M}_{1}$ and $\mathrm{M}_{4}$ muscarinic receptors as potential treatments for Alzheimer's disease and schizophrenia. Neuropsychiatric Disease and Treatment. 2014;10:183191.

7. Shizophrenia. 2016.

8. Pero JE, Rossi MA, Kelly MJ, et al. Optimization of Novel Azabenzimidazolone mGluR2 PAMs with Respect to LLE and PK Properties and Mitigation of CYP TDI. ACS Med. Chem. Lett. ACS Medicinal Chemistry Letters. 2016;7(3):312-317.

9. Wierońska JM, Acher FC, Sławińska A, et al. The antipsychotic-like effects of the mGlu group III orthosteric agonist, LSP1-2111, involves 5-HT ${ }_{1 \mathrm{~A}}$ signalling. Psychopharmacology. 227(4), 711-725.

10. Sławińska A, Wierońska JM, Stachowicz K, et al. The antipsychoticlike effects of positive allosteric modulators of metabotropic glutamate $\mathrm{mGlu}_{4}$ receptors in rodents. Br J Pharmacol. 2013;169(8):1824-1839.
11. Wierońska JM, Sławińska A, Łasoń-Tyburkiewicz M, et al. The antipsychotic-like effects in rodents of the positive allosteric modulator Lu AF21934 involve 5-HT receptor signaling: mechanistic studies. Psychopharmacology. 2015;232(1):259-273.

12. Nicoletti F, Bockaert J, Collingridge GL, et al. Metabotropic glutamate receptors: From the workbench to the bedside. Neuropharmacology. 2011;60(7-8):1017-1041

13. Rook JM, Noetzel MJ, Pouliot WA, et al. Unique signaling profiles of positive allosteric modulators of metabotropic glutamate receptor subtype 5 determine differences in in vivo activity. Biol Psychiatry. 2013;73(6):501-509.

14. Noetzel MJ, Gregory KJ, Vinson PN, et al. A Novel Metabotropic Glutamate Receptor 5 Positive Allosteric Modulator Acts at a Unique Site and Confers Stimulus Bias to mGlu5 Signaling. Mol Pharmacol. 2013;83(4):835-847.

15. Lindsley CW, Brown HA. Phospholipase D as a Therapeutic Target in Brain Disorders. Neuropsychopharmacology. 2012;37(1): 301-302.

16. Menniti FS, Lindsley CW, Conn PJ, et al. Allosteric Modulators for the Treatment of Schizophrenia: Targeting Glutamatergic Networks. Curr Top Med Chem. 2013;13(1):26-54.

17. Bartolomeis A, Sarappa C, Magara S, et al. Targeting glutamate system for novel antipsychotic approaches: relevance for residual psychotic symptoms and treatment resistant schizophrenia. Eur J Pharmacol. 2012;5:682(1-3):1-11.

18. Cioffi CL. Modulation of NMDA receptor functions as a treatment for schizophrenia. Bioorg Med Chem Lett. 2013;15;23(18):5034-5044. 